and provides a reference for future questions. Some clinics have developed their own standard handouts. Others use handouts from books or computerized travel services.

At the University of Iowa, a secretary uses a standard form (Figure) to obtain basic information from callers induding the patient's age, special medical conditions and allergies, travel itinerary, and vaccine history. This form is given to the travel clinic staff physician who checks off immunizations and medications that might be required. A short text has been written for each of the immunizations and medications and for special medical conditions and diseases. The form is returned to the secretary who prepares a handout by merging the designated texts.

\section{Scientific Meetings and Societies}

Scientific meetings provide an opportunity to share experiences with other travel specialists and to obtain up-to-date information on new epidemics, therapies, and immunizations. The American Society of Tropical Medicine and Hygiene (telephone 703-7901745) sponsors a biennial "Travel Medicine Update" in even-numbered years. The update provides an excellent overview of travel medicine as well as important new information for established travel specialists.
The International Society of Travel Medicine (PO. Box 15060, Atlanta, GA 20222-0060; FAX 404-4884427) was founded at the Second Conference on International Travel Medicine in 1991. This conference provides a broad view of the medical care of travelers and expatriates. Proceedings of the Third Conference, held in A pril 1993, may be obtained from the society.

Many other conferences deal specifically or tangentially with travel medicine. Infectious diseases and microbiological societies often have interesting sections on tropical illnesses.

\section{SUM MARY}

Travel medicine is an exciting and rewarding field. This article has provided an overview of basic sources of information. The interested clinician will find many other sources of information on special topics or on travelers with special medical conditions.

\section{REFERENCE}

1. Kozarsky P, Hilton E, Marcus L, Sawyer L, Behrens R Starting and Operating a Travel Clinic. Travel Medicine 2: Proceedings of the Second Conference on International Travel Medicine, Atlanta, GA, May 9-12. 1991. Lobel HO, Steffen R, Kozarsky PE, eds. Atlanta, GA: International Society of Travel Medicine, 1991:326327.

\title{
Inability of Retroviral Tests to Identify Persons with Chronic Fatigue Syndrome
}

\section{by Gina Pugliese, RN, MS Medical News Editor}

The CDC reported the first controlled, blinded trial to examine the ability of retroviral tests (ie, PCR assay, PCR modified assay, and culture for foamy cell cytopathic effect) to distinguish chronic fatigue syndrome (CFS) case patients from controls. None of the assays could differentiate between case patients and controls in either of the two study populations. The findings of this study do not support the hypothesized association between infec- tion with retroviruses and CFS. CFS is characterized by prolonged, debilitating fatigue. Although the cause is unknown, CDC and other researchers have been investigating whether infection with a previously unidentified retrovirus might be an etiologic factor. Based on reports suggesting that retroviral infection with a human T-lymphotrophic virus type II (HTLV-II)like retrovirus or a spumavirus might be associated with CFS, some researchers and commercial laboratories developed assays to test specimens from persons with CFS.
Although previously unidentified retroviral agents might be etiologic factors or cofactors for CFS, the CDC concluded that no scientific basis exists for the use of retroviral testing to confirm the diagnosis of CFS. The CDC recommends that diagnostic testing of patients with suspected CFS should be done solely to exclude other diagnoses.

FROM : Centers for Disease Control and Prevention. Inability of retroviral tests to identify persons with chronic fatigue syndrome, 1992. MMWR. 1993;42:183, 189-190. 
